\title{
Prospective, Randomized Comparisons of Induction of Anesthesia with Ketamine, Propofol and Sevoflurane for Quality of Recovery from Short Sevoflurane Anesthesia in Pediatric Patients
}

\author{
Shin Nakayama, Hajime Furukawa, Hiromune Yanai \\ Department of Anesthesiology, Iwaki Kyoritsu General Hospital, Iwaki, Fukushima, Japan \\ Email: snakayama@md.tsukuba.ac.jp
}

Received 22 June 2015; accepted 19 July 2015; published 22 July 2015

Copyright (C) 2015 by authors and Scientific Research Publishing Inc.

This work is licensed under the Creative Commons Attribution International License (CC BY).

http://creativecommons.org/licenses/by/4.0/

(c) (i) Open Access

\section{Abstract}

Background: Emergence agitation (EA) after sevoflurane anesthesia is common in children. When rapid intravenous induction of general anesthesia is indicated in a brief procedure, the induction agent can reduce the incidence of EA after sevoflurane anesthesia. The aim of this study was to compare the efficacy of intravenous induction with ketamine and propofol for reducing EA in children after short sevoflurane anesthesia. Methods: Children aged 2 to 6 years who were scheduled to undergo inguinal hernia repair were randomly divided into 3 groups to receive $2 \mathrm{mg} / \mathrm{kg}$ ketamine iv, $3 \mathrm{mg} / \mathrm{kg}$ propofol iv, or inspired concentration of $8 \%$ sevoflurane for induction of anesthesia. After a laryngeal mask airway (LMA) insertion, a caudal block was performed in all children. Anesthesia was maintained with $1.5 \%$ sevoflurane and $65 \%$ nitrous oxide in oxygen with spontaneous ventilation. The recovery characteristics were recorded and EA were evaluated by using the Pediatric Anesthesia Emergence Delirium (PAED) scale. Results: One hundred and twenty children were enrolled and randomized to treatment. Children who received ketamine induction had higher incidence of EA than those who received propofol $(42 \%$ vs $16 \%, P<0.05)$ and showed delayed recovery ( $32 \pm 9 \mathrm{~min}$ ) as compared with those who received propofol or sevoflurane $(22 \pm 8 \mathrm{~min}$ and $20 \pm 7 \mathrm{~min}$, respectively, $P<0.05)$. The mean peak PAED score was significantly lower in children who received propofol induction $(6.8 \pm 4.0, P<0.05)$ than ketamine $(11.8$ $\pm 4.1)$ or sevoflurane $(11.6 \pm 3.8)$. Conclusions: Intravenous induction with ketamine does not prevent the incidence of EA and delays recovery. Induction with propofol improves the quality of recovery by reducing the incidence of $E A$ and provides a safe and early recovery. 
Keywords

Pediatric Anesthesia, Emergence Agitation, Sevoflurane, Propofol, Ketamine

\section{Introduction}

Sevoflurane anesthesia has been associated with an increased incidence of emergence agitation (EA) in preschool age children [1] [2]. EA may result in physical harm to the child and caregivers, and may prolong the recovery period if sedative medications are used [3].

When rapid intravenous induction of general anesthesia is indicated in children who have a preexisting intravenous cannula or those who need a rapid tracheal intubation because of a full stomach, the induction agent used should have minimal postoperative side effects to ensure rapid recovery from anesthesia particularly in a brief surgical procedure.

Ketamine is one of the induction agents that produce minimal cardiovascular or respiratory depression. A recent meta-analysis has shown that ketamine has a prophylactic effect in preventing sevoflurane-related EA [4]. However, few studies have investigated the possible effect of intravenous induction with ketamine on the recovery profiles of sevoflurane anesthesia. The aim of this study was to compare the recovery profiles after intravenous induction with ketamine, propofol and inhalation induction with sevoflurane as control in children undergoing short surgical procedures with anesthesia maintained by sevoflurane.

\section{Materials and Methods}

After obtaining institutional committee approval and informed parental consent, 120 children aged 2 to 6 years scheduled to undergo inguinal hernia repair under general anesthesia with caudal block were prospectively studied between April 2006 and March 2007 at Iwaki Kyoritsu General Hospital. All the children were American Society of Anesthesiologists' physical status I and were received oral midazolam premedication $(0.3 \mathrm{mg} / \mathrm{kg}$, maximum $10 \mathrm{mg}$ ). Exclusion criteria included any known allergy to the study drugs and failure of caudal block. Prior to entering the operating room, an iv catheter was inserted in all children.

On entering the operating room, routine monitoring was applied. Children were randomly assigned using a computer-generated allocation to 1 of 3 anesthetic induction groups: (1) group $K(\mathrm{n}=40)$ received $2 \mathrm{mg} / \mathrm{kg}$ ketamine, (2) group $\mathrm{P}(\mathrm{n}=40)$ received $3 \mathrm{mg} / \mathrm{kg}$ propofol, and (3) group $\mathrm{S}(\mathrm{n}=40)$ received inhaled induction with $8 \%$ sevoflurane via a facemask. In group P, $10 \mathrm{mg}$ lidocaine iv was used to minimize the pain on injection with propofol. In all groups, manual ventilation was continued with $8 \%$ sevoflurane and $100 \%$ oxygen. After the insertion of a laryngeal mask airway (LMA), sevoflurane was reduced to $1.5 \%$ and maintained with $65 \%$ nitrous oxide in oxygen. The children maintained spontaneous breathing with assisted ventilation to limit the end-tidal $\mathrm{CO}_{2}$ value of $40 \pm 5 \mathrm{~mm} \mathrm{Hg}$. The caudal block was performed with $0.8 \mathrm{~mL} / \mathrm{kg}$ of $1 \%$ lidocaine. In all cases, the time interval between caudal block placement and skin incision was more than $10 \mathrm{~min}$. If within $60 \mathrm{~s}$ of skin incision, the children's heart rate increased by more than $20 \%$ of the preincision values at the beginning of the surgery, then the block was considered a failure. At the conclusion of the surgery, the anesthetics were discontinued and replaced with 100\% oxygen. When spontaneous breathing was adequate, the LMA was removed in the operating room (time "0" of the recovery period). Subsequently, all children received 100\% oxygen by facemask. The children were discharged from the operating room when they satisfied the following criteria: stable vital signs, patent airway without any manipulation, and oxygen saturation $>95 \%$ of oxygen in $3 \mathrm{~L} / \mathrm{min}$ flow by facemask. The children were then transferred to the recovery room. In the recovery room, all children were given oxygen by facemask, and the oxygen saturation was monitored continuously. Children were left undisturbed, with the exception of calling out their names every minute until they first opened their eyes. After the children were awake, they were examined for a functional block. Children with lower extremity weakness and an absence of response to toe pinch were considered to have an effective caudal block. The children could be transferred to the ward only if they were fully awake, able to breathe deeply, and could maintain oxygen saturation greater than $95 \%$ in room air. All children who reported pain during the recovery period or those who required any analgesics within $1 \mathrm{~h}$ after returning to the ward were excluded from the study.

The following time intervals were recorded: duration of surgery; time of anesthesia (from the start of induc- 
tion to the discontinuation of anesthesia); emergence time (from the end of surgery to spontaneous eye opening without stimulus); and time spent in the recovery room. During recovery, an independent anesthesiologist who was blinded to the anesthetic induction method recorded all the observations and measurements. The children's behavior before the induction of anesthesia was rated on a four-point scale: $1=$ calm; $2=$ not calm, but could be easily calmed; 3 = not easily calmed, moderately agitated or restless; and 4 = combative, excited, or disoriented. This score was used to calculate the incidence of preoperative anxiety, where scores 1 or 2 were considered no anxiety, and scores 3 or 4 were considered presence of anxiety. The children's behaviors were assessed 5, 10, 30, and 60 min after the removal of the LMA. The Pediatric Anesthesia Emergence Delirium (PAED) scale was used to assess EA [5]. EA on the PAED scale was defined as a score $>12$ at any time in the 60 min after the removal of the LMA [6]. The incidence of adverse events such as laryngospasm, breath holding at induction, hypoxemia (oxygen saturation $<96 \%$ ), and vomiting in the operating room or the recovery room were noted. Approximately $24 \mathrm{~h}$ after children returned to the ward, the same blinded anesthesiologist also assessed the incidence of vomiting, presence of nightmares, and any other behavioral changes.

Based on a literature review, we calculated the sample size to detect a reduction in the incidence of agitation from an expected $45 \%$ in the control group with sevoflurane alone to $15 \%$ in the ketamine or propofol group. If the reduction of $30 \%$ in the incidence of agitation was considered clinically important, a sample size of 36 patients in each group would be sufficient to get a two-tailed type I error of 0.05 and a power of $80 \%$. We expected some exclusion from the protocol and increased this number to 40 . One-way analysis of variance and chi-square test or Fisher's exact test as appropriate was used for statistical analysis. When a significant difference was identified, it was followed by an unpaired Student's $t$ test with Bonferroni correction to adjust for multiple comparisons. A $P$ value $<0.05$ was considered significant. Data were presented as mean \pm SD or percentage (\%).

\section{Results}

We enrolled 120 children in this study. Four children (2 in group K, 1 in group P, and 1 in group S) who demonstrated a heart rate response to skin incision were excluded from the study. Two children (1 in group $\mathrm{P}$ and 1 in group S) who showed positive withdrawal to toe pinch when examined in the recovery room were also excluded.

There were no significant differences among the 3 groups in terms of age, gender distribution, weight, duration of surgery, and anesthesia (Table 1). The incidence of preoperative anxiety before the induction of anesthesia was similar among the 3 groups (group $K=34 \%$, group $P=39 \%$, group $S=42 \%$ ).

Table 2 shows the time taken to achieve various events during recovery. The time to eye opening was significantly different among the 3 groups (group K: $25 \pm 11$ min; group P: $17 \pm 8$ min; group S $11 \pm 5$ min, $P<0.05$ ). Children in group K required more time to be discharged from the recovery room. ( $32 \pm 9 \mathrm{~min}$ ) as compared with those who received propofol or sevoflurane (22 \pm 8 min and $20 \pm 7$ min, respectively, $P<0.05$ ).

The severity of EA assessed by the peak PAED scale was significantly lower in group $P(6.8 \pm 4.0, P<0.05)$ than in group K (11.8 \pm 4.1$)$ or group $S(11.6 \pm 3.8)$. Duration of EA was comparable among the 3 groups. Figure 1 shows the percentage of children with EA. At 5 and 10 min after surgery, the incidence of EA in group $\mathrm{S}$ was significantly higher $(47 \%, P<0.05)$ than that in group $\mathrm{K}(16 \%)$ or group $\mathrm{P}(18 \%)$. The incidence of EA in group $\mathrm{K}$ increased gradually in the recovery room. At $30 \mathrm{~min}$ after surgery, the incidence of agitation in group $\mathrm{K}$ (42\%) and group S (39\%) was significantly higher than that in group P $(16 \%, P<0.05)$. Children in group P had significantly lower incidence of EA during all recovery periods. At 60 min after surgery, no difference was observed in the incidence of agitation among the 3 groups.

\begin{tabular}{lccc}
\multicolumn{4}{l}{ Table 1. Patient demographics and anesthetic data. } \\
\hline \multicolumn{1}{c}{ Group } & $\mathrm{K}$ & $\mathrm{P}$ & $\mathrm{S}$ \\
\hline Age (y) & $4 \pm 1$ & $4 \pm 1$ & $4 \pm 1$ \\
Weight (kg) & $15.4 \pm 5.4$ & $15.3 \pm 6.1$ & $15.5 \pm 4.1$ \\
Gender (M/F) & $27 / 11$ & $22 / 16$ & $26 / 12$ \\
Duration of anesthesia (min) & $56 \pm 11$ & $55 \pm 14$ & $56 \pm 15$ \\
Duration of surgery (min) & $34 \pm 10$ & $34 \pm 12$ & $36 \pm 13$ \\
\hline
\end{tabular}

Values are mean \pm SD or numbers. No significant difference. 
Adverse events during perioperative and recovery period are summarized in Table 3. One child in group K developed intraoperative laryngospasm immediately after receiving ketamine. The incidence of breath holding during induction was comparable among the 3 groups. Although none of the children showed apnea, $<96 \%$ oxygen saturation occurred in 7 children in group $\mathrm{K}(18 \%, P<0.05)$ as compared with 2 children in group $\mathrm{P}$ (5\%) and 1 child in group S (3\%) in the recovery room. The incidence of postoperative vomiting was comparable among the 3 groups. Serious complications such as deep sedation or respiratory depression did not occur in the ward. Nightmare and hallucination were not experienced by any of the patients during their hospital stay.

\section{Discussion}

In this study, intravenous induction with $2 \mathrm{mg} / \mathrm{kg}$ ketamine failed to reduce the incidence of EA and delayed recovery and was associated with a higher incidence of hypoxemia in children after short sevoflurane anesthesia.

Table 2. Recovery data.

\begin{tabular}{lccc}
\hline \multicolumn{1}{c}{ Group } & $\mathrm{K}$ & $\mathrm{P}$ & $\mathrm{S}$ \\
\hline Peak PAED scale & $11.8 \pm 4.1$ & $6.8 \pm 4.0^{*}$ & $11.6 \pm 3.8$ \\
Duration of agitation (min) & $17.2 \pm 7.3$ & $15.2 \pm 6.5$ & $15.0 \pm 5.5$ \\
Gender (M/F) & $27 / 11$ & $22 / 16$ & $26 / 12$ \\
Time to discharge from the operating room (min) & $10 \pm 5$ & $8 \pm 3$ & $8 \pm 4$ \\
Time to eye opening (min) & $25 \pm 11^{* *}$ & $17 \pm 8 \dagger$ & $11 \pm 5$ \\
Time to discharge from the recovery room (min) & $32 \pm 9^{* *}$ & $22 \pm 8$ & $20 \pm 7$ \\
\hline
\end{tabular}

Values are mean \pm SD. $* P<0.05$ versus Group K and Group S. $* * P<0.05$ versus Group P and Group $\mathrm{S} . \dagger P<$ 0.05 versus Group $\mathrm{S}$.

Table 3. Incidence of adverse events.

\begin{tabular}{lccc}
\hline \multicolumn{1}{c}{ Group } & K & P & S \\
\hline Laryngospasm (operating room) & $1(3 \%)$ & 0 & 0 \\
Breath holding at induction & $1(3 \%)$ & 0 & $2(5 \%)$ \\
Oxygen saturation <96\% (recovery room) & $7(18 \%)^{*}$ & $2(5 \%)$ & $1(3 \%)$ \\
Vomiting (recovery room) & $4(11 \%)$ & $3(8 \%)$ & $4(11 \%)$ \\
\hline
\end{tabular}

Data are presented as number of patients (proportion). ${ }^{*} P<0.05$ versus group $\mathrm{P}$ and group $\mathrm{S}$.

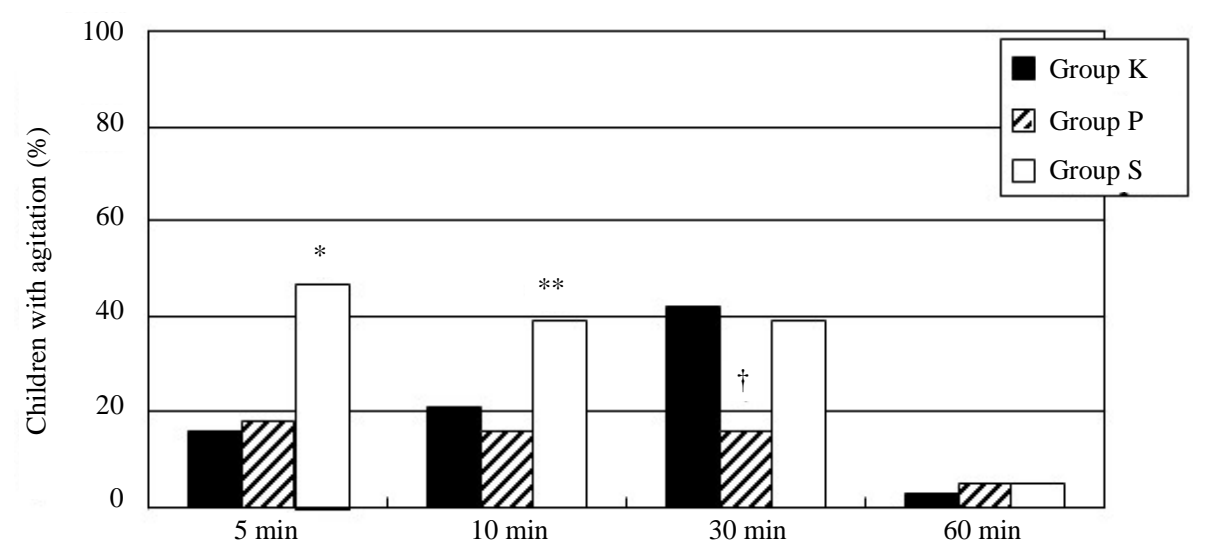

Figure 1. The percentage of children with agitation at each of the time points evaluated. $* P<$ 0.05 versus group $\mathrm{K}$ and group $\mathrm{P}$. $* * P<0.05$ versus group $\mathrm{P} . \dagger P<0.05$ versus group $\mathrm{S}$ and group $\mathrm{K}$. 
Thus, our results indicate that the induction with ketamine does not improve the quality of recovery from sevoflurane anesthesia. In contrast, induction with $3 \mathrm{mg} / \mathrm{kg}$ propofol reduced the incidence of EA and provided a safe and early recovery.

EA after sevoflurane anesthesia has been frequently observed in children, yet the etiology remains unclear [1] [7]. Several factors, such as pain, emergence time, age, premedication, presence of preoperative anxiety, or a variety of perioperative medications have been shown to contribute to the incidence of EA and may complicate the assessment of the emergence behavior. Pain has been recognized as a major factor of EA in children [8]. However, a clear relationship between pain and EA has not been established [3]. Several studies have shown that the incidence of EA after sevoflurane was high even in the presumably pain-free children [2] [7] [9]. In this study, adequate analgesia with caudal block was provided to all children to eliminate pain. Our results also confirmed that the incidence of EA was high in the pain-free children anesthetized with sevoflurane alone. Furthermore, the incidence of EA was similarly high in children received ketamine despite its analgesic effect.

The intravenous induction with ketamine failed to reduce the incidence of EA, yet the standard induction dose was used [10]. The initial distribution half-life of this single iv dose of ketamine is approximately $11 \mathrm{~min}$ and the elimination half-life is about 2.0 to $3.0 \mathrm{~h}$ [10]. The dose of ketamine used in the present study was high enough to delay recovery following short sevoflurane anesthesia. The residual sedative effects of ketamine could explain the lower incidence of EA during the early recovery time, because this slower awakening would have led the observers to grade the agitation with a lower score. However, it should be noted that the timing of assessment of the emergence behavior is important since agitation is never observed when children are still asleep. Thus, repeated assessments in this study revealed a higher incidence of EA in the ketamine group despite the slower awakening. A study comparing the incidence of EA in children after short sevoflurane anesthesia with induction of $5 \mathrm{mg} / \mathrm{kg}$ thiopental and $1.5 \mathrm{mg} / \mathrm{kg}$ of ketamine showed no difference [11]. The incidence of EA in the study was comparable to our results, suggesting anesthetic induction with standard dose of ketamine is not suitable for reducing EA.

Subanesthetic smaller dose of ketamine on emergence may have positive effect on the mood of the child. In adult study, small dose of ketamine has been shown to produce a high feeling of euphoria, and thus appeared to be anxiolytic, which has been suggested to be a result of a property of the N-methyl-D-aspartate (NMDA) receptor antagonist [12]. In children, continuous infusion of ketamine $1 \mathrm{mg} / \mathrm{kg} / \mathrm{h}$ during sevoflurane anesthesia has been shown to reduce the incidence of EA [13] [14]. Furthermore, several studies have shown that a single small-dose of ketamine $(0.25 \mathrm{mg} / \mathrm{kg})$ just before discontinuing anesthesia reduced the incidence of EA without a delay in recovery [15] [16]. The sedative effect of ketamine during emergence can decrease the incidence of EA in children [4]. If smaller dose of ketamine, e.g. less than $1 \mathrm{mg} / \mathrm{kg}$ was used in the present study, the incidence of EA might have been reduced.

The studies on the use of propofol in children have consistently shown to be associated with a low incidence of EA [9] [17] [18]. Our results also confirmed the association of propofol and a reduced incidence of EA. We administered $3 \mathrm{mg} / \mathrm{kg}$ of propofol for induction of anesthesia because the dose of $2.8 \mathrm{mg} / \mathrm{kg}$ was required for loss of eyelash reflex and tolerance of facemask in 90\% of unpremedicated children [19]. The initial distribution half-life of propofol is about $2 \mathrm{~min}$ and the elimination half-life is about $26 \mathrm{~min}$ [20]. The early recovery observed in the propofol group can be explained by the difference of the elimination half-life between propofol and ketamine. Despite the rapid recovery, induction with propofol provided calm awakening and was associated with fewer complications. Thus, when rapid intravenous induction is indicated, propofol would be suitable following short sevoflurane anesthesia.

Our study has a number of limitations. The comparability between the different induction agents regarding the depth of anesthesia remains uncertain. Although we were unable to use an objective monitor of the central nervous system, we used the clinical signs to maintain a comparable level of anesthesia in the 3 groups. Another limitation was that we were unable to demonstrate whether the time spent the hospital was prolonged due to the induction agents used, since according to our hospital regulation all children were admitted to the hospital on the day of the surgery.

\section{Conclusion}

Anesthetic induction with ketamine is ineffective in the prevention of EA in children after short sevoflurane anesthesia. In contrast, anesthetic induction with propofol is effective in the prevention of EA and improves recovery. 


\section{Conflict of Interest}

Shin Nakayama, Hajime Furukawa, Hiromune Yanai have no conflicts of interest.

\section{References}

[1] Veyckemans, F. (2001) Excitation Phenomena during Sevoflurane Anaesthesia in Children. Current Opinion Anaesthesioyl, 14, 339-343. http://dx.doi.org/10.1097/00001503-200106000-00010

[2] Aono, J., Ueda, W., Mamiya, K., Takimoto, E. and Manabe, M. (1997) Greater Incidence of Delirium during Recovery from Sevoflurane Anesthesia in Preschool Boys. Anesthesiology, 87, 1298-1300. http://dx.doi.org/10.1097/00000542-199712000-00006

[3] Voepel-Lewis, T., Malviya, S. and Tait, A.R. (2003) A Prospective Cohort Study of Emergence Agitation in the Pediatric Postanesthesia Care Unit. Anesthesia \& Analgesia, 96, 1625-1630. http://dx.doi.org/10.1213/01.ANE.0000062522.21048.61

[4] Dahmani, S., Stany, I., Brasher, C., Lejeune, C., Bruneau, B., Wood, C., et al. (2010) Pharmacological Prevention of Sevoflurane- and Desflurane-Related Emergence Agitation in Children: A Meta-Analysis of Published Studies. British Journal of Anaesthesia, 104, 216-223. http://dx.doi.org/10.1093/bja/aep376

[5] Sikich, N. and Lerman, J. (2004) Development and Psychometric Evaluation of the Pediatric Anesthesia Emergence Delirium Scale. Anesthesiology, 100, 1138-1145. http://dx.doi.org/10.1097/00000542-200405000-00015

[6] Bajwa, S.A., Costi, D. and Cyna, A.M. (2010) A Comparison of Emergence Delirium Scales Following General Anesthesia in Children. Paediatric Anaesthesia, 20, 704-711. http://dx.doi.org/10.1111/j.1460-9592.2010.03328.x

[7] Cravero, J., Surgenor, S. and Whalen, K. (2000) Emergence Agitation in Paediatric Patients after Sevoflurane Anaesthesia and No Surgery: A Comparison with Halothane. Paediatric Anaesthesia, 10, 419-424. http://dx.doi.org/10.1046/j.1460-9592.2000.00560.x

[8] Davis, P.J., Greenberg, J.A., Gendelman, M. and Fertal, K. (1999) Recovery Characteristics of Sevoflurane and Halothane in Preschool-Aged Children Undergoing Bilateral Myringotomy and Pressure Equalization Tube Insertion. Anesthesia \& Analgesia, 88, 34-38. http://dx.doi.org/10.1097/00000539-199901000-00007

[9] Uezono, S., Goto, T., Terui, K., Ichinose, F., Ishguro, Y., Nakata, Y., et al. (2000) Emergence Agitation after Sevoflurane versus Propofol in Pediatric Patients. Anesthesia \& Analgesia, 91, 563-566. http://dx.doi.org/10.1213/00000539-200009000-00012

[10] Coté, C.J., Lugo, R.A. and Ward, R.M. (2001) Pharmacokinetics and Pharmacology of Drugs in Children. In: Cote, C.J., Todres, I.D., Goudsouzian, N.G. and Ryan, J.F., A Practice of Anesthesia for Infants and Children, 3rd Edition, Saunders, Philadelphia, 121-171.

[11] Jung, H.J., Kim, J.B., Im, K.S., Oh, S.H. and Lee, J.M. (2010) Effect of Ketamine versus Thiopental Sodium Anesthetic Induction and a Small Dose of Fentanyl on Emergence Agitation after Sevoflurane Anesthesia in Children Undergoing Brief Ophthalmic Surgery. Korean Journal of Anesthesiology, 58, 148-152. http://dx.doi.org/10.4097/kjae.2010.58.2.148

[12] Krystal, J.H., Karper, L.P., Seibyl, J.P., Freeman, G.K., Delaney, R., Bremner, J.D., et al. (1994) Subanesthetic Effects of the Noncompetitive NMDA Antagonist, Ketamine, in Humans. Psychotomimetic, Perceptual, Cognitive, and Neuroendocrine Responses. Archives General Psychiatry, 51, 199-214. http://dx.doi.org/10.1001/archpsyc.1994.03950030035004

[13] Kawaraguchi, Y., Miyamoto, Y., Fukumitsu, K., Taniguchi, A., Hirao, O., Kitamura, S., et al. (2002) The Effect of Ketamine on Reducing Postoperative Agitation after Sevoflurane Anesthesia in Pediatric Strabismus Surgery. Masui, 51, 1343-1348.

[14] Chen, J.Y., Jia, J.E., Liu, T.J., Qin, M.J. and Li, W.X. (2013) Comparison of the Effects of Dexmedetomidine, Ketamine, and Placebo on Emergence Agitation after Strabismus Surgery in Children. Canadian Journal of Anesthesia, 60, 385-392. http://dx.doi.org/10.1007/s12630-013-9886-X

[15] Dalens, B.J., Pinard, A.M., Letourneau, D., Albert, N.T. and Truchon, R.J. (2006) Prevention of Emergence Agitation after Sevoflurane Anesthesia for Pediatric Cerebral Resonance Imaging by Small Doses of Ketamine or Nalbuphine Administered Just before Discontinuing Anesthesia. Anesthesia \& Analgesia, 102, 1056-1061. http://dx.doi.org/10.1213/01.ane.0000200282.38041.1f

[16] Abu-Shahwan, I. and Chowdary, K. (2007) Ketamine Is Effective in Decreasing the Incidence of Emergence Agitation in Children Undergoing Dental Repair under Sevoflurane General Anesthesia. Paediatric Anaesthesia, 17, 846-850. http://dx.doi.org/10.1111/j.1460-9592.2007.02298.x

[17] Cohen, I.T., Finkel, J.C., Hannallah, R.S., Hummer, K.A. and Patel, K.M. (2003) Rapid Emergence Does Not Explain Agitation Following Sevoflurane anaesthesia in Infants and Children: A Comparison with Propofol. Paediatric Anaes- 
thesia, 13, 63-67. http://dx.doi.org/10.1046/j.1460-9592.2003.00948.x

[18] OzdemirKol, I., Egilmez, H., Kaygusuz, K., Gursoy, S. and Mimaroglu, C. (2008) Open-Label, Prospective, Randomized Comparison of Propofol and Sevoflurane for Laryngeal Mask Anesthesia for Magnetic Resonance Imaging in Pediatric Patients. Clinical Therapeutics, 30, 175-181. http://dx.doi.org/10.1016/j.clinthera.2008.01.008

[19] Patel, D.K., Keeling, P.A., Newman, G.B. and Radford, P. (1998) Induction Dose of Propofol in Children. Anaesthesia, 43, 949-952. http://dx.doi.org/10.1111/j.1365-2044.1988.tb05659.x

[20] Murat, I., Billard, V., Vernois, J., Zaouter, M., Marsol, P., Souron, R., et al. (1996) Pharmacokinetics of Propofol after a Single Dose in Children Aged 1 - 3 Years with minor burns. Comparison of Three Data Analysis Approaches. Anesthesiology, 84, 526-532. http://dx.doi.org/10.1097/00000542-199603000-00006

\section{Appendix}

The Consolidated Standards of Reporting Trials flow diagram of the randomized trial.

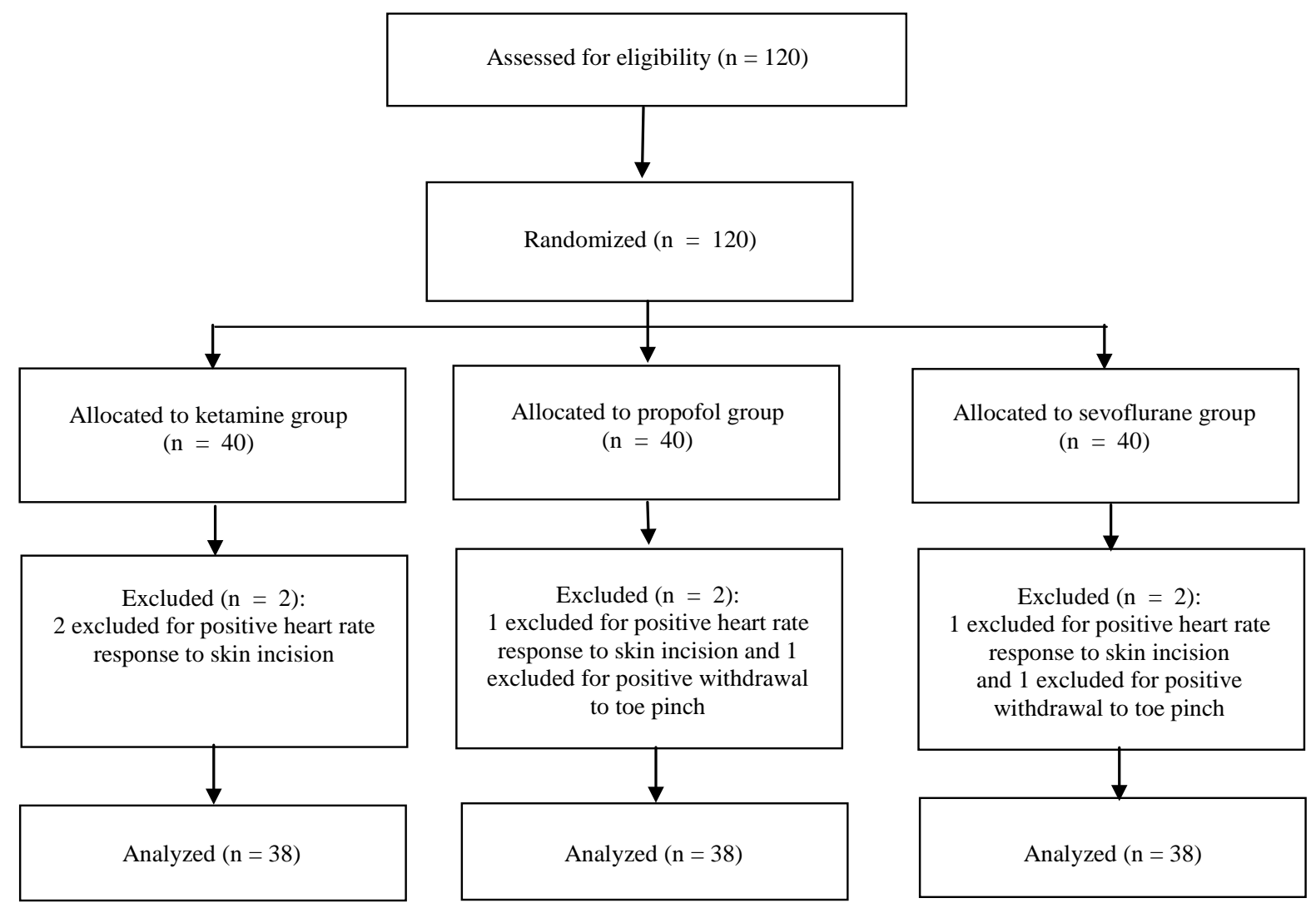

